Article

\title{
Is Gravity Entropic Force?
}

\author{
Rongjia Yang \\ College of Physical Science and Technology, Hebei University, Baoding 071002, China; \\ E-Mail: yangrongjia@tsinghua.org.cn
}

Received: 5 June 2014 ; in revised form: 4 August 2014 / Accepted: 4 August 2014 /

Published: 11 August 2014

\begin{abstract}
If we assume that the source of thermodynamic system, $\rho$ and $p$, are also the source of gravity, then either thermal quantities, such as entropy, temperature, and chemical potential, can induce gravitational effects, or gravity can induce thermal effects. We find that gravity can be seen as entropic force only for systems with constant temperature and zero chemical potential. The case for Newtonian approximation is discussed.
\end{abstract}

Keywords: gravity; thermodynamics; entropic force

\section{Introduction}

It has been recognized that there are profound connections between gravity and thermodynamics [1-5]. Since then, these connections has been steadily becoming stronger. It has been shown that the entropy $S$ can be taken as the Noether charge associated with the diffeomorphism invariance of the theory [6,7]. In [8], the Einstein equation has been derived from the first law of thermodynamics. This attempt also has been investigated in modified gravity theories [9-11] and has been revisited in [12]. In a wide class of spacetime, the field equations in both general relativity and Lovelock theories can be expressed as a thermodynamic identity near the horizon [13-15] (see a review [16]). In [17], the generalized Tolman-Oppenheimer-Volkoff equation is derived by using the maximum entropy principle to a charged perfect fluid, impling that there are fundamental relationships between general relativity and ordinary thermodynamics. The equations of motion for modified gravity theories, such as $F(R)$ gravity, the scalar-Gauss-Bonnet gravity, $F(\mathcal{G})$ gravity, and the non-local gravity, are equivalent to the Clausius relation in thermodynamics [18]. In [19], the Einstein-Hilbert action can be constructed by minimizing the free energy. It was argued that the variation of the surface term evaluated on any null surface which acts a local Rindler horizon can be given a direct thermodynamic 
interpretation [20]. Gravity was explained as an entropic force caused by changes in the information associated with the positions of material bodies [21]. Possible modifications and extensions to this interesting idea were proposed [22-25]. All these studies were based on some assumptions, such as Unruh temperature, horizon, null surfaces, and so on. In [26], The thermal entropy density has been obtained for any arbitrary spacetime without assuming a temperature or a horizon, implying that gravity possesses thermal effects, or, thermal entropy density possesses effects of gravity.

Here we generalize the results in [26] to the case of nonzero chemical potential. The results we obtained indicate that the changes of temperature, entropy, particles, and chemical potential will result in gravitational force, or gravitational force will induce changes of temperature, entropy, particles, and chemical potential.

\section{Relations between Gravity and Thermodynamics}

Both the energy density $\rho$ and the pressure $p$ play important roles in general relativity or thermodynamics. $\rho$ and $p$ are components of the stress-energy tensor in general relativity and are fundamental variables in thermodynamics. Let us begin with the first law of thermodynamics for fluids consisting of particles in curved spacetime

$$
d E=T d S-p d V+\mu d N
$$

where $E, S$, and $N$ represent the total energy, entropy and particle number within the volume $V, \mu$, $T$, and $p$ are the chemical potential, the temperature, and the pressure of the perfect fluid, respectively. $d V=\sqrt{h} d^{3} x$ with $\sqrt{h}$ the determinant of the spatial metric. We take $c=G=1$ and use metric signature $(-,+,+,+)$ throughout this paper. Rewriting (1) in terms of densities

$$
d(\rho V)=T d(s V)-p d V+\mu d(n V),
$$

we can easily get

$$
\rho d V+V d \rho=T V d s+T s d V-p d V+n \mu d V+V \mu d n
$$

where $s$ is the entropy density and $n$ the particle number density. Applying Equation (1) to a unit volume, we have

$$
d \rho=T d s+\mu d n .
$$

Combining Equations (3) and (4), we obtain the integrated form of Gibbs-Duhem relation [27]

$$
T s=\rho+P-\mu n .
$$

For $\mu=0$, Equation (5) reduces to the thermal entropy density obtained in [26]. Now, considering the Einstein equation in [28]

$$
R_{\mu \nu}-\frac{1}{2} g_{\mu \nu} R+\Lambda g_{\mu \nu}=8 \pi T_{\mu \nu}
$$

and the stress energy tensor of the perfect fluid

$$
T_{\mu \nu}=g_{\mu \nu} p+(\rho+p) u_{\mu} u_{\nu}
$$


we obtain

$$
R-4 \Lambda=-8 \pi(3 p-\rho)
$$

Using the $3+1$ decomposition of Einstein equation, we derive [28,29]

$$
n^{\mu} n^{\nu} R_{\mu \nu}+\frac{1}{2} R-\Lambda=8 \pi \mathcal{E},
$$

where $n^{\mu}$ is the unit normal vector field to the 3 dimension hypersurfaces $\Sigma$ and $\mathcal{E}=\Gamma^{2}(\rho+p)-p$ with $\Gamma$ the Lorentz factor. According to the scalar Gauss relation, one can get

$$
\mathcal{R}+K^{2}-K_{i j} K^{i j}-2 \Lambda=16 \pi \mathcal{E},
$$

where $\mathcal{R}$ is the Ricci scalar of the 3 dimension hypersurfaces $\Sigma, K_{i j}$ the extrinsic curvature tensor of $\Sigma$, and $K$ the trace of the $K_{i j}$. Combining Equations (8) and (10), we obtain the expression of $\rho+p$ in general relativity [26]

$$
\rho+p=\frac{1}{4 \pi\left(4 \Gamma^{2}-1\right)}\left[\mathcal{R}+K^{2}-K_{i j} K^{i j}-\frac{1}{2} R\right] .
$$

The four dimension Ricci scalar, $R$, can be decomposed as [29]

$$
R=\mathcal{R}+K^{2}+K_{i j} K^{i j}-\frac{2}{N} \mathcal{L}_{m} K-\frac{2}{N} D_{i} D^{i} N
$$

where $\mathcal{L}_{m}$ is the Lie derivative along $\mathbf{m}$ of any vector tangent to $\Sigma$ and $D_{i}$ is the Levi-Civita connection associated with the metric of the 3 dimension hypersurfaces $\Sigma$. Then Equation (11) can be expressed with three dimension spacial geometrical quantities as [26]

$$
\rho+p=\frac{1}{8 \pi\left(4 \Gamma^{2}-1\right)}\left[\mathcal{R}+K^{2}-3 K_{i j} K^{i j}+\frac{2}{N} \mathcal{L}_{m} K+\frac{2}{N} D_{i} D^{i} N\right] .
$$

In order to relate thermodynamics with gravity, we must suggest a hypothesis. We first review Newton's equivalence principle so as to have a better understanding. When a particle falls freely in a gravitational field, the gravity is also the inertial force. This fact leads to Newton's equivalence principle that the inertial and the gravitational mass of a particle are equal. Like the case of Newton's equivalence principle, for any perfect fluid in spacetime the energy density $\rho$ and the pressure $p$ are both the sources of gravity and thermodynamic system. So we put forward a hypothesis that the source of thermodynamic system, $\rho$ and $p$, are also the source of gravity, namely

$$
(\rho+p)_{\text {gravitational source }}=(\rho+p)_{\text {thermal source }}
$$

For radiation, this hypothesis holds [26]. In [8], the $\delta Q$ of thermodynamic system was assumed as the energy flux and then the Einstein equation was obtained. Analogous assumptions have also been suggested in $[17,26,30,31]$, though these assumptions have not been aware of by the authors. With this hypothesis (14) and combining Equations (5) and (13), we obtain

$$
T s+\mu n=\frac{1}{8 \pi\left(4 \Gamma^{2}-1\right)} \times\left[\mathcal{R}+K^{2}-3 K_{i j} K^{i j}+\frac{2}{N} \mathcal{L}_{m} K+\frac{2}{N} D_{i} D^{i} N\right] .
$$


The terms of the left-hand side of this equation are thermodynamical quantities, while the terms of the right-hand side of this equation are geometrical quantities of the spacetime. Equation (15) implies that gravity can induce thermal effects, or, thermal quantities, such as entropy, temperature, and chemical potential, can induce gravitational effects. To understand the physical significance of Equation (15) well, we consider the Newtonian approximation: $g_{\mu \nu}=\eta_{\mu \nu}+h_{\mu \nu}$, with $h_{\mu \nu} \ll 1$. For matter with $p \simeq 0$ (the pressure of a body becomes important when its constituent particles are traveling at speeds close to that of light, which we can exclude from the Newtonian limit by hypothesis), such as dust or dark matter, we have the Poisson equation $\nabla^{2} \varphi=4 \pi \rho$ with $\varphi=-h_{00} / 2$. Taking into account Gibbs-Duhem relation (5) and the hypothesis (14), we obtain

$$
\nabla^{2} \varphi=4 \pi(T s+\mu n)
$$

Equation (16) (or (15)) relates gravity with thermodynamics. Variations in temperature, entropy, chemical potential, and particle number will lead to a variation in the potential $\varphi$, and vice versa. Equation (16) (or (15)) also indicates that gravity is related to the entropy but is not entropic force. Only for systems with constant temperature and zero chemical potential, gravitational force is entropic force. These results confirm the arguments in [32] that experiments with ultra-cold neutrons in the gravitational field of Earth disprove the speculations on the entropic origin of gravitation. In [33], it also argued that the argument for the entropic origin of gravity is problematic.

\section{Conclusions}

We have shown that if we conjecture that the source of thermodynamic system, $\rho$ and $p$, are also the

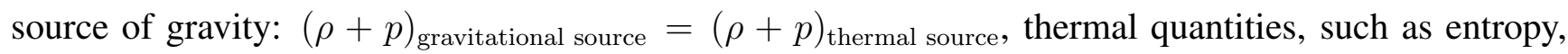
temperature, and chemical potential, can induce gravitational effects, or gravity can induce thermal effects. For Newtonian approximation, the gravitational potential is related to the temperature, entropy, chemical potential, and particle number, which implies that gravity is entropic force only for systems with constant temperature and zero chemical potential. For general case, gravity is not an entropic force. Whether the results obtained here can be generalized to the case of modified gravity, such as $F(R)$ gravity [10] and $F(\mathcal{G})$ gravity [18], is worthy of investigation. All the analyses have been carried out without assuming a specific expression of temperature or horizon. For a static system at thermal equilibrium in general relativity, the temperature of the perfect fluid may take the form, $T \sqrt{-g_{00}}=$ const., which is called the Tolman temperature [34-36]. Whether the temperature in Equation (1) can be taken as Tolman temperature is also worthy of further investigation. The results we obtained confirm that there is a profound connection between gravity and thermodynamics.

\section{Acknowledgments}

This study is supported in part by National Natural Science Foundation of China (Grant Nos. 11147028 and 11273010) and Hebei Provincial Natural Science Foundation of China (Grant No. A2014201068). 


\section{Conflicts of Interest}

The author declares no conflict of interest.

\section{References}

1. Cocke, W.J. A maximum entropy principle in general relativity and the stability of fluid spheres. Ann. Inst. Henri Poincaré 1965, 2, 283-306.

2. Bekenstein, J.D. Black holes and entropy. Phys. Rev. D 1973, 7, 2333-2346.

3. Hawking, S.W. Particle Creation by Black Holes. Commun. Math. Phys. 1975, 43, 199-220.

4. Davies, P.C.W. Scalar particle production in Schwarzschild and Rindler metrics. J. Phys. A 1975, 8, 609-616.

5. Unruh, W.G. Notes on black hole evaporation. Phys. Rev. D 1976, 14, doi:10.1103/PhysRevD.14.870.

6. Wald, R.M. Black hole entropy is the Noether charge. Phys. Rev. D 1993, 48, 3427-3431.

7. Iyer, V.; Wald, R.M. Some properties of Noether charge and a proposal for dynamical black hole entropy. Phys. Rev. D 1994, 50, 846-864.

8. Jacobson, T. Thermodynamics of Spacetime: The Einstein Equation of State. Phys. Rev. Lett. 1995, 75, 1260-1263.

9. Eling, C.; Guedens, R.; Jacobson, T. Non-equilibrium thermodynamics of spacetime. Phys. Rev. Lett. 2006, 96121301.

10. Elizalde, E.; Silva, P.J. $F(R)$ gravity equation of state. Phys. Rev. D 2008, 78, 061501.

11. Brustein, R.; Hadad, M. The Einstein equations for generalized theories of gravity and the thermodynamic relation delta $Q=T \delta S$ are equivalent. Phys. Rev. Lett. 2009, 103, 101301.

12. Makela, J.; Peltola, A. Gravitation and thermodynamics: The Einstein equation of state revisited. Int. J. Mod. Phys. D 2009, 18, 669-689.

13. Padmanabhan, T. Classical and quantum thermodynamics of horizons in spherically symmetric space-times. Class. Quan. Gravity 2002, 19, 5387-5408.

14. Paranjape, A.; Sarkar, S.; Padmanabhan, T. Thermodynamic route to field equations in Lancos-Lovelock gravity. Phys. Rev. D 2006, 74, 104015.

15. Kothawala, D.; Sarkar, S.; Padmanabhan, T. Einstein's equations as a thermodynamic identity: The Cases of stationary axisymmetric horizons and evolving spherically symmetric horizons. Phys. Lett. B 2007, 652, 338-342.

16. Padmanabhan, T. Thermodynamical Aspects of Gravity: New insights. Rep. Prog. Phys. 2010, 73, 046901.

17. Gao, S. A general maximum entropy principle for self-gravitating perfect fluid. Phys. Rev. D 2011, 84, 104023.

18. Bamba, K.; Geng, C.Q.; Nojiri, S.; Odintsov, S.D. Equivalence of modified gravity equation to the Clausius relation. Europhys. Lett. 2010, 89, 50003.

19. Bracken, P. The Einstein-Hilbert Action Horizons and Connections with Thermodynamics. Adv. Stud. Theor. Phys. 2012, 6, 83-93.

20. Parattu, K.; Majhi, B.R.; Padmanabhan, T. Structure of the gravitational action and its relation with horizon thermodynamics and emergent gravity paradigm. Phys. Rev. D 2013, 87, 124011. 
21. Verlinde, E.P. On the Origin of Gravity and the Laws of Newton. J. High Energy Phys. 2011, 4, doi:10.1007/JHEP04(2011)029.

22. Gao, C. Modified Entropic Force. Phys. Rev. D 2010, 81, 087306.

23. Li, M.; Wang Y. Quantum UV/IR Relations and Holographic Dark Energy from Entropic Force. Phys. Lett. B 2010, 687, 243-247.

24. Cai, Y.-F.; Saridakis, E.N. Inflation in Entropic Cosmology: Primordial Perturbations and non-Gaussianities. Phys. Lett. B 2011, 697, 280-287.

25. Hendi, S.H.; Sheykhi, A. Entropic Corrections to Einstein Equations. Phys. Rev. D 2011, 83, 084012.

26. Yang, R. The thermal entropy density of spacetime. Entropy 2013, 15, 156-161.

27. Gao, S.; Wald, R. Physical process version of the first law and the generalized second law for charged and rotating black holes. Phys. Rev. D 2001, 64, 084020.

28. Wald, R.M. General Relativity; The University of Chicago Press: Chicago, IL, USA, 1984.

29. Gourgoulhon, E. 3+1 Formalism and Bases of Numerical Relativity. 2007, arXiv:gr-qc/0703035.

30. Fang, X.; Gao, S. General proof of the entropy principle for self-gravitating fluid in static spacetimes. 2013, arXiv:1311.6899.

31. Green, S.R.; Schiffrin, J.S.; Wald, R.M. Dynamic and Thermodynamic Stability of Relativistic, Perfect Fluid Stars. Class. Quantum Gravity 2014, 31, 035023.

32. Kobakhidze, A. Gravity is not an entropic force. Phys. Rev. D 2011, 83, 021502.

33. Gao, S. Is gravity an entropic force. Entropy 2011, 13, 936-948.

34. Tolman, R.C. On the Weight of Heat and Thermal Equilibrium in General Relativity. Phys. Rev. 1930, 35, doi:10.1103/PhysRev.35.904904.

35. Tolman, R.C.; Ehrenfest, P. Temperature Equilibrium in a Static Gravitational Field. Phys. Rev. 1930, 36, doi:10.1103/PhysRev.36.1791.

36. Rovelli, C.; Smerlak, M. Thermal time and the Tolman-Ehrenfest effect: Temperature as the "speed of time". Class. Quantum Gravity 2011, 28, 075007.

(c) 2014 by the author; licensee MDPI, Basel, Switzerland. This article is an open access article distributed under the terms and conditions of the Creative Commons Attribution license (http://creativecommons.org/licenses/by/3.0/). 